\title{
Effect of Mulligan's mobilization with movement technique on gait function in stroke patients
}

\author{
SAng-Lim Kim, PT, MSc ${ }^{1)}$, Byoung-Hee Lee, PT, $\mathrm{PhD}^{2)^{*}}$ \\ 1) Graduate School of Physical Therapy, Sahmyook University, Republic of Korea \\ 2) Department of Physical Therapy, Sahmyook University: 815 Hwarang-ro, Nowon-gu, Seoul 01795, \\ Republic of Korea
}

\begin{abstract}
Purpose] We examined the effectiveness of Mulligan's mobilization with movement (MWM) technique on spatiotemporal variables of gait in individuals who had a stroke. [Subjects and Methods] Twenty-four subjects were randomly divided into 2 groups: Mulligan's mobilization with movement group ( $\mathrm{n}=12)$ and "weightbearing with placebo" mobilization with movement group $(n=12)$. The subjects in the mobilization with movement group performed 5 sets of 10 glides a day, 5 times a week for 4 weeks. The mobilization with movement technique comprised grade III movements that involved gliding and resting. The control group subjects performed lunges in the same conditions as those of the experimental group. Gait function was measured in terms of spatiotemporal parameters to determine the effect of mobilization with movement. [Results] The mobilization with movement group showed significant improvements in velocity, cadence, stride length, single-support time, and step length of the affected side, and step length and stride length of the non-affected side. Overall, the mobilization with movement group showed significantly greater improvements than the control group in terms of velocity, cadence, and singlesupport time of the affected side. [Conclusion] Mobilization with movement can be used to improve the gait function of patients recovering from stroke.

Key words: Stroke rehabilitation, Mulligan's mobilization with movement, Gait function
\end{abstract}

(This article was submitted Mar. 1, 2016, and was accepted May 14, 2016)

\section{INTRODUCTION}

Flaccidity appears during the early stage after stroke; however, as time passes, it is accompanied by spasticity, which occurs in $90 \%$ of stroke patients. It limits voluntary movements because of muscle stiffness. In particular, spasticity of the ankle plantar flexor disrupts static and dynamic balance, as well as the normal gait pattern ${ }^{1,2}$. Shortened calf muscles in individuals with hemiparesis cause ankle joint stiffness, increase its hypomobility and resistance to mobility, and decrease passive joint mobility, which ultimately causes limitation of the ankle joint ${ }^{3,4)}$. Abnormal muscle tension and movement limitation due to muscle weakness of the ankle joint cause limitation of functional activities such as changing from a sitting position to standing, locomotion, and climbing stairs ${ }^{5}$. Stroke patients have difficulty in standing up without help, and difficulties in negotiating obstacles start to emerge during walking ${ }^{6}$. One of the reasons for the decrease in gait speed and asymmetric gait in hemiplegic patients is the limitation of passive and active movements due to structural changes in the connective tissue and articular capsule around the ankle joint ${ }^{7}$. The motor function of the ankle joint greatly affects balance and walking abilities. In addition, improvement of walking ability is a major goal in patients undergoing stroke rehabilitation ${ }^{8)}$.

Manual therapy is widely used and advocated for many aspects of peripheral joint dysfunction. Mulligan's mobilization with movement (MWM) technique is commonly used in musculoskeletal physiotherapy. The recently developed manual therapy techniques include Brian Mulligan's widely used MWM for peripheral joint pain, which has been shown to improve range of motion and promote earlier return to function after lateral ankle sprain ${ }^{9,10)}$. The clinical efficacy of Mulligan's

*Corresponding author. Byoung-Hee Lee (E-mail: 3679@syu.ac.kr)

(C2016 The Society of Physical Therapy Science. Published by IPEC Inc.

This is an open-access article distributed under the terms of the Creative Commons Attribution Non-Commercial No Derivatives (by-nc-nd) License $<$ http://creativecommons.org/licenses/by-nc-nd/4.0/>. 
MWM technique is also applicable to the MWM technique for talocrural dorsiflexion, which is frequently used to improve talocrural dorsiflexion deficits that often occur after lateral ankle sprain ${ }^{10)}$. MWMs correct this by repositioning the joint, causing it to track normally ${ }^{11)}$. The mechanisms behind the effectiveness of MWMs are based on mechanical dysfunction and therefore on positional fault correction ${ }^{12}$.

Thus, the purpose of this study was to verify whether Mulligan's MWM technique improve the temporal and spatial variables of the gait of individuals who had a stroke.

\section{SUBJECTS AND METHODS}

The study participants were chosen from among 24 stroke patients who were undergoing physical therapy at a rehabilitation hospital. The participant selection criteria were as follows: those who had hemiparesis due to stroke with onset at $>6$ months, the ability to follow verbal instructions, the ability to communicate at a certain level, recovered the motor functions of their paretic lower limbs at Brunnstrom stage 2-4, and a score of $>24$ points on the Mini-Mental State Examination-Korean. Patients were excluded if they had a neurological condition, orthopedic disease, or visual impairment. All the patients who participated in this trial provided their written informed consent after receiving a full explanation of the expected results and side effects of the intervention. The present study was approved by the Sahmyook University Institutional Review Board (SYUIRB2015-114).

The 24 subjects were randomly assigned to either Mulligan's MWM group (MWM; $n=12$ ) or a "weight-bearing with placebo" MWM group (control; $n=12$ ). The general characteristics of the 12 subjects in the experimental group were as follows: 6 males and 6 females; a mean age of 41.58 years; a mean height of $165.67 \mathrm{~cm}$; a mean weight of $62.42 \mathrm{~kg}$; and a mean time since stroke onset of 8.87 months; right- and left-side hemiplegia in 8 and 4 cases, respectively. The characteristics of the 12 subjects in the control group were as follows: 6 males and 6 females; a mean age of 53.0 years; a mean height of $164.58 \mathrm{~cm}$; a mean weight of $60.67 \mathrm{~kg}$; and a mean time since stroke onset of 10.46 months; and right- and left-side hemiplegia in 5 and 7 cases, respectively. No significant differences in these characteristics were found between the 2 groups.

The participants in the experimental group performed Mulligan's MWM technique at 5 sets of 10 glides a day, 5 times a week for 4 weeks. The participants in the control group performed lunges in the same conditions as the experimental group. The participants in the control group were trained for 5 sets of 10 lunges a day, 5 times a week for 4 weeks. Before the intervention, all the participants received conventional physical therapy for 30 minutes per day, 5 days per week over a 4-week period. Gait ability was blindly assessed before and after the intervention.

Mulligan's MWM technique consisted of gliding and resting. Each gliding involved 10 seconds of posterior talar glide with dorsiflexion and 5 seconds of resting between the gliding. For the gliding, grade III movements were used. The therapist applied a sustained posteroanterior glide to the tibia through the belt by leaning backward while the talus and forefoot were fixed in the space between the thumb and the second finger of the right hand. The other hand was positioned anteriorly over the proximal tibia to direct the knee over the line of the second and third toes. Then, the participant was instructed to perform a slow dorsiflexion movement until the first onset of pain or end of range of motion without the heel lifting off the couch.

Gait function was measured by using GAITRite (GAITRite, CIR system Inc., Havertown, Pennsylvania, USA). GAITRite was used for the measurement of spatiotemporal parameters, including gait velocity, cadence, step length, and stride length ${ }^{13)}$. The measurement was performed 3 times. To eliminate fatigue from walking, the subjects took a 30 -second rest after walking each time.

The SPSS 18.0 software program (SPSS Inc., Chicago, IL, USA) was used for statistical analysis. The data showed normal distribution as assessed by the Kolmogorov-Smirnov test. A paired t-test was used to compare pretest and posttest measurements of gait function within the groups, and the independent t-test was used to compare the difference in gait function before and after training between the groups. A p value of $<0.05$ was considered statistically significant.

\section{RESULTS}

The MWM group showed significant improvements in velocity, which increased from $41.35 \mathrm{~cm} / \mathrm{s}$ before training to $51.72 \mathrm{~cm} / \mathrm{s}$ after training $(\mathrm{p}<0.001)$; cadence, step length, stride length, and single-support time of the affected side; and step length and stride length of the non-affected side $(\mathrm{p}<0.05)$. In addition, the control group demonstrated significant improvements in step length and stride length of the non-affected side $(\mathrm{p}<0.05)$. The MWM group showed significantly greater improvements than the control group in terms of velocity, cadence, and single-support time of the affected side $(\mathrm{p}<0.05$; Table 1).

\section{DISCUSSION}

This study was conducted to assess the effects of Mulligan's MWM techniques on the temporal and spatial variables of gait of individuals who had a stroke. The application of Mulligan's MWM techniques effectively improved the gait function of the patients in this study.

The pressure from weight bearing on the affected side of the lower extremity is decreased by up to $43 \%$, which makes 
Table 1. Comparison of gait ability within and between the groups $(n=24)$

\begin{tabular}{|c|c|c|c|c|c|c|}
\hline \multirow{3}{*}{ Parameters } & \multicolumn{4}{|c|}{ Values } & \multicolumn{2}{|c|}{ Change values } \\
\hline & \multicolumn{2}{|c|}{ MWM (n=12) } & \multicolumn{2}{|c|}{ Control $(n=12)$} & \multirow{2}{*}{$\begin{array}{c}\text { MWM ( } \mathrm{n}=12) \\
\text { Before-after }\end{array}$} & \multirow{2}{*}{$\begin{array}{c}\text { Control }(\mathrm{n}=12) \\
\text { Before-after }\end{array}$} \\
\hline & Before & After & Before & After & & \\
\hline Velocity $(\mathrm{cm} / \mathrm{s})$ & $41.35(14.73)$ & $51.72(15.42)^{* *}$ & $49.69(23.26)$ & $51.55(22.20)$ & $10.37(6.63)^{\dagger}$ & $1.87(7.13)$ \\
\hline Cadence (step/min) & $70.25(17.90)$ & $78.50(14.59)^{*}$ & $78.45(21.57)$ & $76.50(19.04)$ & $8.25(8.50)^{\dagger}$ & $-1.95(7.71)$ \\
\hline A: step length $(\mathrm{cm})$ & $36.35(6.28)$ & $41.33(7.04)^{*}$ & $35.80(8.49)$ & $38.36(9.79)$ & $4.97(5.53)$ & $2.56(5.49)$ \\
\hline A: stride length $(\mathrm{cm})$ & $69.90(13.77)$ & $78.14(13.54)^{*}$ & $73.72(17.52)$ & $78.45(17.58)$ & $8.23(7.93)$ & $4.72(8.77)$ \\
\hline A: single-support time $(\% / G C)$ & $20.08(5.81)$ & $23.33(4.55)^{*}$ & $24.00(6.53)$ & $23.20(7.19)$ & $3.25(4.79)^{\dagger}$ & $-2.51(1.87)$ \\
\hline NA: step length $(\mathrm{cm})$ & $33.17(8.60)$ & $36.57(7.95)^{*}$ & $36.31(9.11)$ & $39.43(8.33)^{*}$ & $3.40(3.38)$ & $3.11(4.86)$ \\
\hline NA: stride length $(\mathrm{cm})$ & $69.60(13.56)$ & $77.92(13.32)^{*}$ & $72.63(15.92)$ & $77.64(17.22)^{*}$ & $8.32(8.03)$ & $5.01(7.32)$ \\
\hline
\end{tabular}

Data are presented as means (SD); ${ }^{*} \mathrm{p}<0.05,{ }^{* *} \mathrm{p}<0.001$ : significant difference within each group; ${ }^{\dagger} \mathrm{p}<0.05$ : significant difference between the groups; MWM: Mulligan's mobilization with movement group; A: affected side; NA: non-affected side

stroke patients prone to falls to the affected side because of loss of balance. They experience difficulty in standing without assistance, which impairs their ambulatory abilities ${ }^{6)}$. For these reasons, gait and balance training is essential for stroke patients. This training involves exercise of the ankle to improve gait and balance. In particular, problems with the dorsiflexors of the ankle were reported to be the highest risk factor of falls ${ }^{14}$, and tonic paralysis in the plantar flexors of the ankle disrupt the normal gait pattern ${ }^{15}, 16$. Some treatments are available to resolve these problems, including neurodevelopmental techniques, functional electrical stimulation, biofeedback, strength training, and treadmill training. However, in this study, the efficacy of Mulligan's MWM technique was verified. This technique consisted of gliding and resting. Each gliding consisted of 10 seconds of posterior talar glide with dorsiflexion of the ankle joint and 5 seconds of resting between the gliding. For the gliding, grade III movements were used. The therapist applied a sustained posteroanterior glide to the tibia through the belt by leaning backward while the talus and forefoot were fixed in the space between the thumb and the second right-hand finger. The MWM group had significantly greater improvements in velocity, cadence, and single-support time of the affected side $(p<0.05)$ than the control group, which suggests that Mulligan's MWM technique could improve the gait ability of patients recovering from stroke.

The symptoms of postural imbalance in stroke patients are evident during standing because of the increased weight bearing toward the non-paretic side. Decreased standing balance and loss of the ability to shift weight toward the paretic leg can also develop after a stroke ${ }^{17}$. Difficulty in shifting weight toward the paretic side can affect gait function. Therefore, limitations in movements can decrease the range and amount of physical activities that can be performed and could lead to further decreases in postural balance, which may ultimately result in the deterioration of physical health ${ }^{18)}$. Therefore, this study investigated a sustained posteroanterior glide to the tibia for improvement of weight shift in stroke patients. The MWM group showed a significant improvement in single-support time of the affected side, which increased from $20.08 \%$ /gait cycle (GC) before training to $23.33 \% / \mathrm{GC}$ after training $(\mathrm{p}<0.05)$. For increasing the single-support time of the affected side, Mulligan's MWM is more effective than the lunge position. Muscle cooperation in the ankle joint puts the center of gravity on the ankle joint in the standing position. It requires normal range of motion of the ankle joint and muscular strength ${ }^{19,20)}$. The normal range of motion of the ankle joint in the standing position improved in the MWM group owing to the posteroanterior glide to the tibia.

Based on the findings of the present study, Mulligan's MWM could increase the velocity, cadence, step length, stride length, and single-support time of the affected side, and the step length and stride length of the non-affected side. Moreover, increased single-support time of the affected side will positively influence the motivation of patients during GC and weight bearing on the non-paretic side toward maintaining their bodies within the limits of stability. Thus, the positive effect of Mulligan's MWM on gait function and its effectiveness as a treatment regimen for improving the GC in stroke patients were confirmed.

This study has limitations, including the lack of diversity in the dependent variables. This is because only gait ability was measured. In addition, Mulligan's MWM technique is commonly used in musculoskeletal physical therapy; however, it was used in this study to improve the GC in stroke patients. Hence, further research on the efficacy of Mulligan's MWM in stroke rehabilitation should be conducted.

\section{REFERENCES}

1) Thibaut A, Chatelle C, Ziegler E, et al.: Spasticity after stroke: physiology, assessment and treatment. Brain Inj, 2013, 27: 1093-1105. [Medline] [CrossRef]

2) Yom C, Cho HY, Lee B: Effects of virtual reality-based ankle exercise on the dynamic balance, muscle tone, and gait of stroke patients. J Phys Ther Sci, 2015, 27: 845-849. [Medline] [CrossRef] 
3) Harlaar J, Becher JG, Snijders CJ, et al.: Passive stiffness characteristics of ankle plantar flexors in hemiplegia. Clin Biomech (Bristol, Avon), 2000, 15: 261-270. [Medline] [CrossRef]

4) Given JD, Dewald JP, Rymer WZ: Joint dependent passive stiffness in paretic and contralateral limbs of spastic patients with hemiparetic stroke. J Neurol Neurosurg Psychiatry, 1995, 59: 271-279. [Medline] [CrossRef]

5) Andriacchi TP, Andersson GB, Fermier RW, et al.: A study of lower-limb mechanics during stair-climbing. J Bone Joint Surg Am, 1980, 62: 749-757. [Medline]

6) Campbell FM, Ashburn AM, Pickering RM, et al.: Head and pelvic movements during a dynamic reaching task in sitting: implications for physical therapists Arch Phys Med Rehabil, 2001, 82: 1655-1660. [Medline] [CrossRef]

7) Kluding PM, Santos M: Effects of ankle joint mobilizations in adults poststroke: a pilot study. Arch Phys Med Rehabil, 2008, 89: 449-456. [Medline] [CrossRef]

8) Kim N, Park Y, Lee BH: Effects of community-based virtual reality treadmill training on balance ability in patients with chronic stroke. J Phys Ther Sci, 2015, 27: 655-658. [Medline] [CrossRef]

9) Mulligan BR: Mobilizations with movement. J Manual Manip Ther, 1993, 1: 154-156. [CrossRef]

10) Vicenzino B, O’Brien T: A study of the effects of Mulligan's mobilization with movement treatment of lateral ankle pain using a case study design. Man Ther, 1998, 3: 78-84. [CrossRef]

11) Wilson E: The Mulligan concept: NAGS, SNAGS and mobilizations with movement. J Bodyw Mov Ther, 2001, 5: 81-89. [CrossRef]

12) Vicenzino B, Paungmali A, Teys P: Mulligan's mobilization-with-movement, positional faults and pain relief: current concepts from a critical review of literature. Man Ther, 2007, 12: 98-108. [Medline] [CrossRef]

13) McDonough AL, Batavia M, Chen FC, et al.: The validity and reliability of the GAITRite system's measurements: a preliminary evaluation. Arch Phys Med Rehabil, 2001, 82: 419-425. [Medline] [CrossRef]

14) Schoenfelder DP, Van Why K: A fall prevention educational program for community dwelling seniors. Public Health Nurs, 1997, 14: $383-390$. [Medline] [CrossRef]

15) Umphred DA, Burton GU, Lazaro RT: Neurological rehabilitation, 5th ed. New York: Elsevier Science Health Science Div, 2007.

16) Jung GU, Moon TH, Park GW, et al.: Use of augmented reality-based training with EMG-triggered functional electric stimulation in stroke rehabilitation. J Phys Ther Sci, 2012, 25: 147-151. [CrossRef]

17) Srivastava A, Taly AB, Gupta A, et al.: Post-stroke balance training: role of force platform with visual feedback technique. J Neurol Sci, 2009, 287: 89-93. [Medline] [CrossRef]

18) Yang S, Hwang WH, Tsai YC, et al.: Improving balance skills in patients who had stroke through virtual reality treadmill training. Am J Phys Med Rehabil, 2011, 90: 969-978. [Medline] [CrossRef]

19) Shumway-Cook A, Woollacott M: Attentional demands and postural control: the effect of sensory context. J Gerontol A Biol Sci Med Sci, 2000 , 55: M10-M16. [Medline] [CrossRef]

20) Park YH, Kim YM, Lee BH: An ankle proprioceptive control program improves balance, gait ability of chronic stroke patients. J Phys Ther Sci, 2013, 25: 1321-1324. [Medline] [CrossRef] 